\title{
Enhanced Radiative Decay Rate of Confined Green Fluorescent Protein in Polyvinylpyrrolidone-based Nanofiber
}

\author{
Sabriye Acikgoz, ${ }^{1, *}$ Yakup Ulusu, ${ }^{2}$ Hasan Yungevis, ${ }^{1}$ Faruk Ozel, ${ }^{1}$ Abdurrahman Özen, ${ }^{1}$ \\ Isa Gokce, ${ }^{3}$ Koray Kara, ${ }^{4}$ Mahmut Kuş ${ }^{4}$ \\ ${ }^{1}$ Department of Material Science and Engineering, Faculty of Engineering, Karamanoğlu \\ Mehmetbey University, 70100 Karaman, Turkey \\ ${ }^{2}$ Department of Bioengineering, Faculty of Engineering, Karamanoğlu Mehmetbey University, \\ 70100 Karaman, Turkey \\ ${ }^{3}$ Department of Bioengineering, Faculty of Engineering, Gaziosmanpaşa University, 60240 \\ Tokat, Turkey \\ ${ }^{4}$ Department of Chemical Engineering, Faculty of Engineering, Selcuk University, 42100 Konya, \\ Turkey
}

\section{Supporting Information}

\section{ProtParam}

User-provided sequence:

\begin{tabular}{|c|c|c|c|c|c|}
\hline $1 \underline{0}$ & $2 \underline{0}$ & $3 \underline{0}$ & $4 \underline{0}$ & $5 \underline{0}$ & $6 \underline{0}$ \\
\hline MHHHHHHSSS & KGEELFTGVV & PILVELDGDV & NGHKFSVSGE & GEGDATYGKL & TLKFICTTGK \\
\hline $7 \underline{0}$ & 80 & $9 \underline{0}$ & 100 & 110 & 120 \\
\hline LPVPWPTLVT $^{-}$ & TFSYGVQCFS & RYPDHMKQHD & FFKSAMPEG $\bar{Y}$ & VQERTIFFKD & DGNYKTRAEV \\
\hline 130 & 140 & 150 & 160 & 170 & 180 \\
\hline KFEGDTLVNR & IELKGIDFKE & DGNILGHKLE & YNYNSHNVYI & MADKQKNGIK & VNFKIRHNIE \\
\hline $19 \underline{0}$ & $20 \underline{0}$ & $21 \underline{0}$ & $22 \underline{0}$ & $23 \underline{0}$ & $24 \underline{0}$ \\
\hline DGSVQLADHY & QQNTPIGDGP & VLLPDNHYLS & TQSALSKDPN & EKRDHMVLLE & FVTAAGITHG \\
\hline
\end{tabular}




\section{COMPUTED PARAMETERS:}

Number of amino acids: 246

Molecular weight: 27883.3

Theoretical pI: 6.03

Amino acid composition:

\begin{tabular}{|c|c|c|}
\hline Ala (A) & 8 & $3.3 \%$ \\
\hline Arg (R) & 6 & $2.4 \%$ \\
\hline Asn (N) & 13 & $5.3 \%$ \\
\hline Asp (D) & 18 & $7.3 \%$ \\
\hline Cys (C) & 2 & $0.8 \%$ \\
\hline Gln (Q) & 8 & $3.3 \%$ \\
\hline Glu (E) & 16 & $6.5 \%$ \\
\hline Gly (G) & 22 & $8.9 \%$ \\
\hline His (H) & 16 & $6.5 \%$ \\
\hline Ile (I) & 12 & $4.9 \%$ \\
\hline Leu (L) & 19 & $7.7 \%$ \\
\hline Lys (K) & 20 & $8.1 \%$ \\
\hline Met (M) & 6 & $2.4 \%$ \\
\hline Phe (F) & 13 & $5.3 \%$ \\
\hline Pro (P) & 10 & $4.1 \%$ \\
\hline Ser (S) & 13 & $5.3 \%$ \\
\hline Thr (T) & 15 & $6.1 \%$ \\
\hline Trp (W) & 1 & $0.4 \%$ \\
\hline Tyr (Y) & 11 & $4.5 \%$ \\
\hline Val (V) & 17 & $6.9 \%$ \\
\hline Pyl (O) & 0 & $0.0 \%$ \\
\hline Sec (U) & 0 & $0.0 \%$ \\
\hline (B) & 0 & $0.0 \%$ \\
\hline$(Z)$ & 0 & $0.0 \%$ \\
\hline$(X)$ & 0 & $0.0 \%$ \\
\hline
\end{tabular}

Total number of negatively charged residues (Asp + Glu): 34

Total number of positively charged residues (Arg + Lys): 26 


\section{Atomic composition:}

$\begin{array}{lcr}\text { Carbon } & \text { C } & 1247 \\ \text { Hydrogen } & \text { H } & 1900 \\ \text { Nitrogen } & \text { N } & 338 \\ \text { Oxygen } & \text { O } & 375 \\ \text { Sulfur } & \text { S } & 8\end{array}$

Formula: $\mathrm{C}_{1247} \mathrm{H}_{1900} \mathrm{~N}_{338} \mathrm{O}_{375} \mathrm{~S}_{8}$

Total number of atoms: 3868

\section{Extinction coefficients:}

Extinction coefficients are in units of $\mathrm{M}^{-1} \mathrm{~cm}^{-1}$, at $280 \mathrm{~nm}$ measured in water.

Ext. coefficient 22015

Abs $0.1 \%(=1 \mathrm{~g} / 1) \quad 0.790$, assuming all pairs of Cys residues form cystines

Ext. coefficient 21890

Abs $0.1 \%(=1 \mathrm{~g} / \mathrm{l}) \quad 0.785$, assuming all Cys residues are reduced

\section{Estimated half-life:}

The N-terminal of the sequence considered is $\mathrm{M}$ (Met).

The estimated half-life is: 30 hours (mammalian reticulocytes, in vitro).

$>20$ hours (yeast, in vivo).

$>10$ hours (Escherichia coli, in vivo).

\section{Instability index:}

The instability index (II) is computed to be 32.49

This classifies the protein as stable.

\footnotetext{
Aliphatic index: 72.44
}

Grand average of hydropathicity (GRAVY): -0.589 